\title{
Successful radiotherapy treatment of lacrimal gland infiltration in patient with Sjögren's syndrome
}

\author{
Petera $\mathrm{J}^{1}$, Sirak $\mathrm{I}^{1}$, Langrova $\mathrm{H}^{2}$, Maisnar $\mathrm{V}^{3}$, Slezak $\mathrm{R}^{4}$, Brokesova $\mathrm{S}^{1}$ \\ Department of Oncology and Radiotherapy, University Hospital Hradec Kralove, Czech Republic. \\ petera@fnhk.cz
}

\begin{abstract}
Objective: To present a single case report on successful radiotherapy treatment of lacrimal gland infiltration in patient with Sjögren's syndrome.

Background: Radiotherapy is occassionally used for the treatment of benign disorders. There is no report on use of radiotherapy for local treatment of the Sjögren's syndrome in the literature.

Methods: Female patient with lacrimal gland involvement as a part of Sjögren's syndrome with diplopia and visus deterioration was treated by radiotherapy with eye shielding.

Results: Regression of the infiltration with full restoration of visus and minimal acute radiation reaction was achieved. Conclusion: A case report of successful use of local radiotherapy in the treatment of lacrimal gland affected by Sjögren's syndrome is presented (Fig. 3, Ref. 6). Full Text in PDF www.elis.sk.

Key words: radiotherapy; lacrimal gland; Sjögren's syndrome.
\end{abstract}

Sjögren's syndrome (SS) is an autoimmune disease of exocrine glands that occurs with a prevalence of $0.5-1 \%$. It has a female predominance and is commonly diagnosed in the 4 th -5 th decades of life. It is characterized by progressive lymphocytic infiltration of exocrine glands and epithelium at multiple sites. Involvement of the lacrimal and salivary glands eventually leads to the 2 typical features of the disease: keratoconjunctivitis sicca and xerostomia. SS may occur in 2 forms: primary, when the clinical manisfestation of the syndrome is seen alone, and secondary, when associated with another autoimmune disease, most commonly rheumatoid arthritis.

Numerous criteria have been proposed to facilitate the accurate classification and diagnosis of SS. The criteria were recently revised and modified by the American-European Consensus Group (1). This set of criteria includes 6 different items: ocular symptoms; oral symptoms; ocular signs; histopatologic examination of minor salivary glans; objective evidence of salivary gland involvement; and serologic evaluation of autoantibodies.

The treatment of patients with Sjögren's syndrome is directed toward the particular areas of the body that are involved, and toward complications, such as infection. There is no report on the use of radiotherapy for local treatment of this disease in the literature.

'Department of Oncology and Radiotherapy, University Hospital Hradec Králové, Czech Republic, ${ }^{2}$ Department of Ophthalmology, University Hospital Hradec Králové, Czech Republic, ${ }^{3}$ Department of Hematology, University Hospital Hradec Králové, Czech Republic, and ${ }^{4}$ Department of Stomatology, University Hospital Hradec Králové, Czech Republic

Address for correspondence: J. Petera, MD, PhD, Department of Oncology and Radiotherapy, University Hospital Hradec Kralove, Sokolska 581, CZ-500 05, Hradec Králové, Czech Republic. Phone: +420.495.832183, Fax: +420.495.832081

Acknowledgments: Research Project 00179906 of the Ministry of Health, Czech Republic

\section{Methods}

Forty-four years old female patient with a tumor of the right parotis underwent a parotidectomy in March 2001. The histology was evaluated as a benign lymphoepithelial lesion - the Mikulicz's disease. Further investigations in cooperation with stomatologist and immunologist led to the diagnosis of Sjögren's syndrome. The patient met 5 of 6 classification criteria of the American-European Consensus Group (1): oral symptoms (dry mouth), ocular signs (Schimers test), histopathology, salivary gland involvement (unstimulated whole salivary flow), antibodies to Ro/SSA/ and La/SSB/ antigen. In November 2001, elevated IgM concentrations were discovered. In May 2002, extirpation of swollen right submandibular gland was performed. Histologic examination showed (again) benign lymphoepithelial lesion.

In June 2007, the patient was examined at the Deptartment of Ophthalmology for swelling of the right lacrimal gland and keratoconjunctivitis sicca. Lubrication therapy was indicated. The lacrimal gland swelling progressed during 3 months accompanied by pains, keratoconjunctivitis, diplopia, and visus deterioration as a result of hypermetropisation caused by enlarged lacrimal gland (Fig. 1). CT examination showed homogenous and enhanced lacrimal gland of $30 \times 16 \times 33 \mathrm{~mm}$. Biopsy of the lacrimal gland proved focal lymphocytic infiltration. Because of progressive lesion the patient was suggested to consider radiotherapy treatment.

The patient had signed an informed consent and radiotherapy was performed between 18th October and 12th November 2007. The right upper eyelid was irradiated by a single radiation field, which was shaped by lead shielding to avoid exposition of surrounding healthy tissues. The eye was covered by leaden eye-bath located under the eyelid (Fig. 2). Therapeutic X-ray machine with beam energy of $180 \mathrm{kV}$ was used for irradiation. The dosis was 15 times 2 Gy with daily fractionation. 


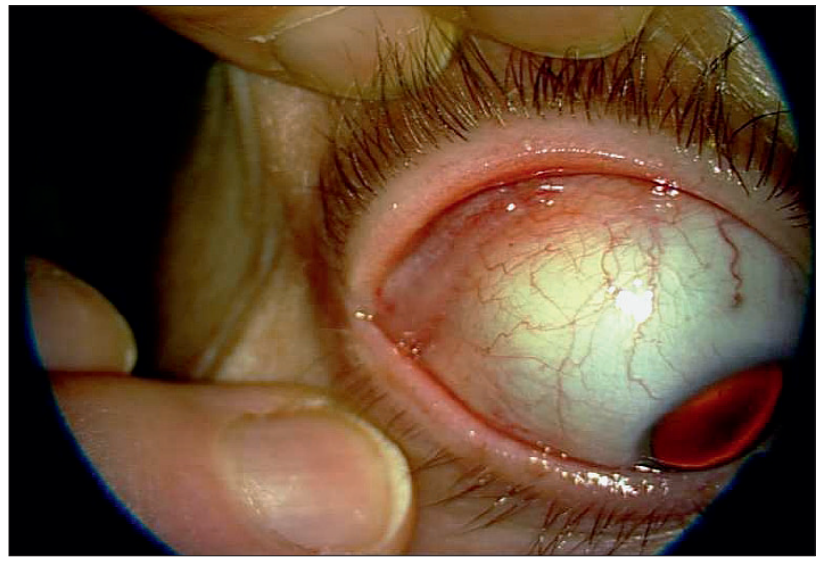

Fig. 1. Local finding before radiotherapy.

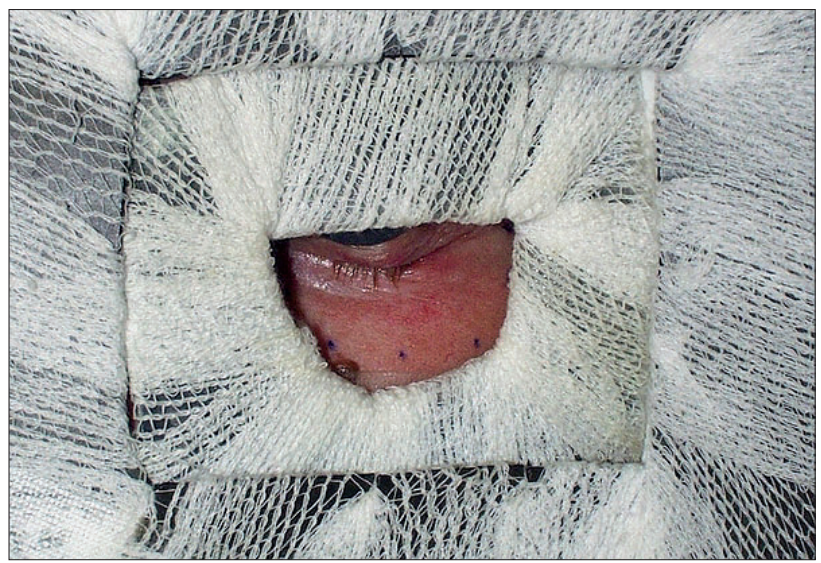

Fig. 2. The technique of radiotherapy.

\section{Results}

By the end of radiotherapy the eyelid swelling and pains had resolved. The acute adverse reaction consisted of transient periocular skin erytema and conjunctive hyperemia. During the ophthalmological examination on the 29th January 2008 the patient presented without diplopia, able to read and to watch television, without lacrimal gland prominence, and with cured acute radiation reaction.

In October 2008, bilateral parotidectomy was performed because of recurrent infiltration of the right parotid and left parotid swelling. Lagophthalmos of the right eye after surgery was solved by temporary tarzoraphy. At the last ophthamological examination in April 2010, the regression of the right eyelid infiltration lasted, and the perimeter after radiotherapy was intact (Fig. 3).

\section{Discussion}

The treatment of primary SS is focused on sicca features and relief of dry eyes and mouth. Systemic medicamentous treatment is based mainly on the administration of steroids, antirheumatic drugs, and immunosuppressants. It has frequent side effects and is used mainly for the treatment of extraglandular manifestations or complications. Patient with symptomatic disease progression of

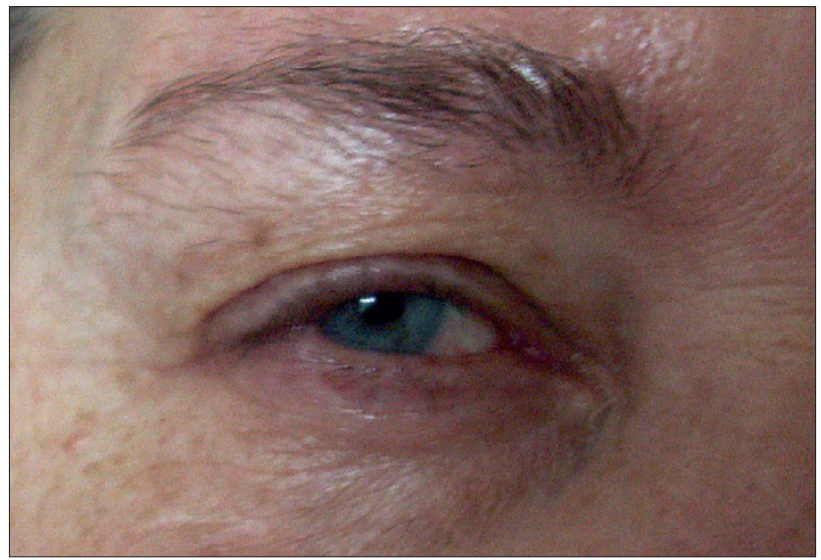

Fig. 3. Local finding 2.5 years after radiotherapy.

lacrimal gland involvement despite standard therapy was referred to our department to consider local radiotherapy.

Antiproliferative effect of radiotherapy is used not only in the treatment of malignant tumors, but also for irradiation of benign diseases including lymphoproliferative disorders like lymphoid orbital pseudotumor $(2,3,4)$ or Kimurá s disease (5). The median dose used for the irradiation is about $30 \mathrm{~Gy}$. There is only one paper dealing with radiotherapy of Mikulicz's disease to be found (6), but no other paper on the use of radiotherapy for SS has been reported up to now.

At our department we use an eye sparing radiotherapy technique for irradiation of skin cancers in the eyelid region with very satisfactory results. We are presenting a case report of successful use of this technique in the treatment of lacrimal gland affected SS.

\section{Conclusion}

We are presenting a case report of successful use of local radiotherapy in the treatment of lacrimal gland affecred by Sjögren's syndrome.

\section{References}

1. Vitali C, Bombardieri S, Jonsson R et al. Classification criteria for Sjögren's syndrome: a revised version of the European criteria proposed by the American-European Consensus Group. Ann Rheum Dis 2002; 61 (6): 554-558.

2. Lanciano R, Fowble B, Sergott R et al. The results of radiotherapy for orbital pseudotumor. Int J Radiat Oncol Biol Phys 1990; 18 (2): 407-411.

3. Keleti D, Flickinger JC, Hobson SR, Mittal BB. Radiotherapy of lymphoproliferative diseases of the orbit. Surveillance of 65 cases. Am J Clin Oncol 1992; 15 (5): 422-427.

4. Wagner W, Gerding H, Busse H. Pseudotumor orbitae - a chameleon in diagnosis and therapy? Strahlenther Onkol 1992; 168 (9): 528-535.

5. Chang AR, Kim K, Kim HJ et al. Outcomes of Kimura's disease after radiotherapy or nonradiotherapeutic treatment modalities. Int J Radiat Oncol Biol Phys 2006; 65 (4): 1233-1239.

6. Hazel J, Bouchard J. The treatment of Mikulicz's disease with radiation therapy. J Can Assoc Radiol 1964; 15: 70-73.

Received July 29, 2010. Accepted January 13, 2012. 